Archives de sciences sociales des religions

134 | avril - juin 2006

Varia

\title{
Helmut Zander, Geschichte der Seelenwanderung in
}

Europa

Darmstadt, Primusverlag, 1999, 869 p.

Bruno Michon

\section{OpenEdition}

\section{Journals}

Édition électronique

URL : http://journals.openedition.org/assr/3657

DOI : 10.4000/assr.3657

ISSN : $1777-5825$

Éditeur

Éditions de l'EHESS

Édition imprimée

Date de publication : 1 mai 2006

Pagination : 147-299

ISBN : 2-7132-2092-0

ISSN : 0335-5985

\section{Référence électronique}

Bruno Michon, «Helmut Zander, Geschichte der Seelenwanderung in Europa », Archives de sciences sociales des religions [En ligne], 134 | avril - juin 2006, document 134-95, mis en ligne le 12 septembre 2006, consulté le 21 septembre 2020. URL : http://journals.openedition.org/assr/3657 ; DOI : https:// doi.org/10.4000/assr.3657

Ce document a été généré automatiquement le 21 septembre 2020

(c) Archives de sciences sociales des religions 


\title{
Helmut Zander, Geschichte der Seelenwanderung in Europa
}

Darmstadt, Primusverlag, 1999, 869 p.

\author{
Bruno Michon
}

1 L'ouvrage d'Helmut Zander nous propose d'entrer dans la longue histoire de la transmigration en Europe. Loin des platitudes sur le succès de l'idée de réincarnation dans l'univers religieux européen, l'auteur de la monumentale «Geschichte der Seelenwanderung in Europa » traverse les siècles à la recherche des traces historiques de métempsycose, révolution et autre palingenèse.

2 Car il s'agit de s'entendre sur le sujet du débat, ce que met particulièrement bien en perspective l'ouvrage de $\mathrm{H}$. Zander. Le concept de "réincarnation» englobe, aujourd'hui, ce que l'on a nommé, dans l'antiquité, «paîingenesi » ou « métempsycos », « Revolutio » chez saint Augustin ou gilgul dans la kabbale juive. L'auteur replace chacun de ces termes dans leurs univers de signification eschatologique propre.

3 Signalons, par ailleurs, que le concept allemand de « Seelenwanderung » ne connait pas d'équivalent français sinon l'expression de «migration des âmes». On pourrait proposer, toutefois, une traduction qui paraît proche du "Seelenwanderung » de $\mathrm{H}$. Zander soit celle de «transmigration ». La notion de réincarnation ne traduit en effet ni l'idée de l'âme, ni celle du mouvement, pourtant centrale dans l'appréhension de la trajectoire post-mortem de celle-ci.

4 Helmut Zander a construit son livre comme une gigantesque trame chronologique qui s'étend des philosophes pré-socratiques aux différents avatars du concept de réincarnation aujourd'hui en Occident. Dans un (trop) court chapitre initial, l'auteur traite de la place de la réincarnation dans les cultures non européennes et, entre autres, asiatiques, il se tourne toutefois promptement vers l'Europe en débutant une longue recherche sur l'Antiquité.

5 L'épineuse question de l'origine véritable du concept de transmigration en Europe est alors évaluée à partir de trois hypothèses : soit celle d'une origine asiatique, ou d'une ancienne tradition locale grecque, ou enfin, d'une tradition non hellénique mais 
géographiquement proche (entre autre Thrace). L'auteur montre, brillamment, que les sources permettent de ne tirer aucune conclusion. Ces hypothèses resteront donc, malheureusement, ce qu'elles sont et la question de l'origine demeure, à l'heure actuelle, irrésolue.

Quoi qu'il en soit, les philosophes et théologiens glosèrent, des siècles durant, sur les différentes possibilités d'une incarnation renouvelée de l'âme. Les pythagoriciens et les néo-platoniciens apportèrent, à cette conception des événements post-mortem, ses lettres de noblesse: ils deviendront une source d'inspiration classique chez les humanistes de la Renaissance.

Cependant l'apparition d'une voie de Salut concurrente, proposée par le christianisme, occultera l'idée de transmigration durant plusieurs siècles. L'auteur conclut ainsi cette seconde partie: «Des problèmes centraux tels celui de la préservation de l'identité dans la migration; celui des possibilités, difficilement calculables, d'une sortie du processus de la réincarnation; ou encore, celui de la menace de la possible incarnation en animal, sont devenus sans objet suite à la théorie chrétienne de la résurrection [...]. À la fin de l'antiquité la notion de transmigration n'était certainement devenue plus que l'ombre d'elle-même: une spéculation issue d'un débat obsolète» (p.119, ma traduction). Cette concurrence n'implique pas nécessairement la disparition de l'idée de transmigration et l'auteur montre dans son troisième chapitre intitulé très justement "L'Occident, la réincarnation en arrière-plan", qu'un grand nombre de mouvements poursuivent la réflexion en ce sens.

8 Il passe, ainsi, en revue le concept de tanassukh de l'islam chiite, ce dernier influencé par les sectes gnostiques d'Asie centrale et par le néo-platonisme ; le concept hébreu de gilgul qui connaîtra un destin florissant, remplissant les pages du Bahir, compilation cabalistique dont la date de composition est incertaine (probablement le $\mathrm{xII}^{\mathrm{e}}$ siècle) ou les spéculations du célèbre cabaliste Isaak Luria; les cathares, par une forme de docétisme gnostique, développent une croyance proche de la transmigration, dans laquelle les âmes « chutent » véritablement du ciel dans un corps physique.

9 L'auteur montre, parfaitement, l'extraordinaire influence des spéculations antiques sur ces différents mouvements. La redécouverte des "Anciens", durant la Renaissance, déclenchera l'intérêt pour la métempsycose, intérêt qui ne cessera de s'accroître jusqu'à nos jours.

10 Ainsi, les noms de Giordano Bruno, Francisais Mercurius van Helmont ou Gottfried Wilhelm Leibniz constituent autant de jalons dans l'histoire de l'expansion de l'idée de réincarnation à l'époque moderne. En concluant, $\mathrm{H}$. Zander montre néanmoins que la réincarnation reste un thème marginal jusqu'à la période contemporaine. L'essor immense de la transmigration au XIXe siècle y trouve, toutefois, ces racines. L'auteur montre dans cette optique que, déjà, les théories modernes de la réincarnation sont forgées à partir du paradigme scientifique/rationaliste - ou du moins, dans un premier temps du paradigme de la " philosophie naturelle »- comme cela sera la norme au XIX siècle. Helmut Zander consacre près de la moitié de son ouvrage au xix siècle. Face à la multiplication des sources, il fait le choix judicieux de se concentrer sur la zone germanique. Les remarques sociohistoriques qu'il formule permettent d'appréhender la lente et progressive diffusion du concept de transmigration d'une couche sociale d'intellectuels nobles à l'ensemble de la population. Il faut, toutefois, attendre la seconde moitié du XIX ${ }^{e}$ siècle pour assister à sa véritable popularisation. 
11 L'écrivain et philosophe Gotthold Ephraïm Lessing, qui dans son Erziehung des Menschengeschlechts, propose une conception véritablement évolutionniste de la transmigration, est paradigmatique de la tournure empruntée par l'idée de réincarnation au début $\mathrm{du} \mathrm{XIX}^{\mathrm{e}}$ siècle. Chez lui, elle devient un véritable système postmortem qui tend vers la réalisation spirituelle de l'individu. Parallèlement, et en partie contre la dichotomie corps/esprit, Johann Gottfried Herder propose l'idée de migration planétaire de l'âme qui connaîtra un succès considérable qui ne se démentira pas jusqu'à nos jours. En outre, une page, peu connue, de la réincarnation en Europe, est son emploi chez les Utopistes du $\mathrm{XIX}^{\mathrm{e}}$ siècle. L'idéal égalitaire d'un Fourrier, par exemple, s'appuie sur celle-ci pour répondre aux apories de la théodicée (les individus mauvais, souvent des industriels capitalistes, seront punis dans leurs prochaines incarnations). On se doute, néanmoins, que l'arrivée triomphale des philosophies et religions orientales en Europe constitua le véritable catalyseur de l'engouement populaire pour la réincarnation.

12 L'auteur revient, bien sûr, sur le rôle précurseur de Arthur Schopenhauer dans cette histoire de la réception du bouddhisme. Le philosophe allemand est, certainement, le premier à intégrer, à ce point, une philosophie orientale dans son système philosophique. Ce sont, toutefois, les milieux ésotériques qui constitueront la véritable base de propagation de la pensée orientale. Ainsi, dès 1875, la société théosophique de la médium polonaise Helena Petrovna Blavatsky, met en place un système de pensée syncrétique, très influencé par le bouddhisme et l'hindouisme et dont les ramifications s'étendent à de nombreux mouvements contemporains. De la société anthroposophique de Rudolf Steiner (dont Helmut Zander est, par ailleurs, un éminent spécialiste) au mouvement du New Age, son influence est capitale pour l'histoire de la transmigration.

13 Pour finir l'auteur aborde quelques mutations contemporaines de l'idée de réincarnation. Sans se départir d'une certaine ironie, il décrit au lecteur les effets les plus surprenants qu'a pu engendrer l'expansion de celle-ci: de la publicité, à la thérapie en passant par les monuments aux morts, H. Zander dresse un panorama édifiant de la place désormais centrale qu'occupe la réincarnation dans le paysage religieux actuel. L'ouvrage se clôt sur un chapitre d'anthropologie historique où l'auteur aborde astucieusement, thème après thème, les résultats de sa recherche historique.

La «Geschichte der Seelenwanderung in Europa » est donc une somme d'érudition historique qui mérite l'attention des sociologues - germanophones dans un premier temps, en attendant une heureuse traduction - qui pourraient avoir tendance à parler trop précipitamment de l'engouement contemporain sur la transmigration. Si engouement il y a aujourd'hui, il s'agit, en effet, de ne pas conclure trop hâtivement quant à l'origine asiatique de l'idée de réincarnation en Europe, ni sur la soumission de celle-ci à l'évolutionnisme européen.

15 La riche histoire de cette idée, dans le cadre géographique européen, montre au contraire qu'il existe un véritable pluralisme philosophique et théologique de la réflexion sur la réincarnation. Toutes les solutions semblent avoir été envisagées au cours des siècles. À côté des nécessaires préalables anthropologiques, que sont le dualisme corps/esprit et l'immortalité de l'âme, se trouvent des idées plus originales comme celle de migration planétaire, de possibilité d'une incarnation en plante ou en animal, de métamorphose plutôt que d'incarnation... La réincarnation fit ainsi l'objet de réflexions des plus rationnelles au plus insolites. S'il fallait, toutefois, relever une 
limite aux acquis de cet ouvrage, ce serait dans le "germanocentrisme» auquel l'auteur s'est, consciemment, limité pour une grande partie de sa recherche. Il serait, en effet, passionnant de prolonger les réflexions de Helmut Zander à d'autres aires géographiques.

16 En outre, et il s'agit là d'un sujet qui me tient particulièrement à cœur, ses développements sur Rudolf Steiner m'ont paru un peu faible quant à sa connaissance du sujet (qui fut l'objet de sa thèse). Rudolf Steiner est, certainement, l'un des penseurs les plus prolixes et les plus respectés de la scène ésotérique européenne en ce qui concerne la transmigration (voire au-delà quant à ses spéculations pédagogiques et médicales). Toute la tension qui existe chez cet auteur, entre une réflexion résolument chrétienne et un concept de réincarnation oscillant entre origines européenne et asiatique, me paraît paradigmatique de ce qui peut exister, aujourd'hui, dans de nombreux nouveaux mouvements religieux, dans le New Age ou dans le channeling. 